\title{
NUCLEAR AND EXTENDED INFRARED EMISSION IN PAIRED AND ISOLATED GALAXIES
}

Roc M. Cutri

Steward Observatory

University of Arizona

\section{INTRODUCTION}

The empirical connection between gravitational and collisional interactions among galaxies and enhanced activity has been well-documented. However, the physical mechanisms which are responsible for triggering the various forms of activity have not been determined. We present the preliminary results of a study of the nuclear and integrated infrared properties of galaxies chosen from the Catalog of Isolated Pairs of Galaxies in the Northern Hemisphere (Karachentsev 1972; hereafter CPG) and the Catalog of Isolated Galaxies (Karachentseva 1973; hereafter KI). Observations of these large, unbiased samples of paired and isolated galaxies are analyzed with the hope of identifying which aspects of galaxy encounters are most closely coupled to the presence of activity.

\section{NUCLEAR PROPERTIES}

Cutri and McAlary (1985) reported that the $10 \mu \mathrm{m}$ luminosity distribution of a sample of CPG galaxies exhibited a high luminosity tail not present in the distribution of a control sample of bright spiral galaxies and Virgo spirals. Although the CPG and control samples had very similar absolute B magnitude distributions, the CPG sample spanned a larger range in redshift space than the non-interacting samples, and it was felt that the result could suffer from a Malmquist bias. Therefore, we have begun to re-examine this relationship by using the excellent performance of the new Multiple Mirror Telescope Bolometer system to obtain sensitive broadband $10.4 \mu \mathrm{m}$ measurements of a sample of $\mathrm{KI}$ galaxies which better match the redshift range of the CPG sample.

The subsets of the CPG and KI for this study were chosen to have $m_{\mathrm{Pr}} \leq 14.0$ or $\mathrm{m}_{\mathrm{tot}} \leq \mathbf{1 3 . 3}$ for close pairs, and $0^{\circ} \leq \delta \leq \mathbf{3 7 ^ { \circ }}$. For the CPG sample, Cutri and McAlary obtained data for pairs with LIN, DIS or ATM classifications (i.e. showing some sign of disturbed morphology). We have begun to carry out new photometry of the non-disturbed (SEP) pairs, as well. To date, approximately $2 / 3$ of the new KI sample has been observed, and the $10 \mu \mathrm{m}$ luminosity distributions of the CPG and KI samples are shown in Figure 1. As before, the CPG sample exhibits a distribution essentially similar to the non-interacting $\mathrm{KI}$ sample, except for a population of sources with $\mathrm{L}_{10}>10^{\circ} \mathrm{L}_{\odot}$. The KI distribution shows several galaxies with higher luminosities than the earlier control samples, but still $<10^{9} \mathrm{~L}_{\odot}$. It should be noted that although they satisfy the isolation criteria of the KI, the two most 
luminous galaxies in the sample, KI 359 (=NGC 2960) and KI 1004 (=NGC 7479), both appear disturbed on POSS plates. The statistical significance of the difference between the two distributions is difficult to assess since the CPG galaxies exhibit a base population similar to that of the KI sample. However, a Gehan two-sample test does reject the hypothesis that the two are drawn from the same parent population at better than the $98 \%$ level.

Figure 2 shows the ratio of the IRAS $12 \mu \mathrm{m}$ flux density to the small aperture $10 \mu \mathrm{m}$ flux density for the CPG and KI galaxies, giving a measure of the extent of the mid-infrared emission. Bearing in mind that the IRAS beam sampled roughly 100 times the area of our small aperture measurements, these data show that wherever nuclear $10 \mu \mathrm{m}$ emission is detected it is strongly concentrated within a diameter of $1 \mathrm{kpc}$. This compactness is consistent with the characteristic sizes of galactic nuclei at $10 \mu \mathrm{m}$ measured by Rieke (1976).

The nuclei of the high luminosity sources from the CPG sample all show HII region or Liner optical spectra, indicating that the elevated luminosities are due to enhanced star formation in and around the nuclear regions. The presence of enhanced nuclear $10 \mu \mathrm{m}$ emission in the interacting systems suggests that mass transfer between galaxies is probably not the mechanism which triggers the activity, because of the difficulty of transporting infalling material directly to the nuclei. Such a conclusion was also reached by Keel et al. (1985), whose proposed that tidally enhanced fueling of the nuclei might lead to the nuclear activity. Finally, we find no obvious dependence in the $10 \mu \mathrm{m}$ luminosity distribution on pair separation, angular size, velocity difference or interaction morphology.

\section{EXTENDED PROPERTIES}

We have examined IRAS ADDSCAN data for a large subset of both the CPG and $\mathrm{KI}$ to investigate galaxy-wide emission in interacting and isolated systems. Because the ADDSCAN processing co-adds all IRAS survey scans covering a source, these data provide the most sensitive survey measurements from the IRAS mission, typically 2-3 times better than the Point Source Catalog. This enables better assessment to be made of the midinfrared (12 and $25 \mu \mathrm{m}$ ) emission, which can contribute significantly to the total infrared luminosity. Furthermore, ADDSCAN source profiles of the CPG galaxies can be examined to determine more accurately whether close pairs of galaxies have been resolved. The magnitude limit of the samples was $m_{\mathrm{pc}} \leq 15.0$ or $\mathrm{m}_{\mathrm{tot}} \leq 14.3$ for close pairs.

The distributions of the integrated 12-100 $\mu \mathrm{m}$ luminosities of the $\mathrm{KI}$ and CPG sample galaxies are shown in Figure 3. The CPG pairs have been divided into two sets: the first being pairs with projected separations smaller than the IRAS beam size (unresolved 
pairs) and those separated widely enough to have been resolved by the satellite. Unlike the nuclear $10 \mu \mathrm{m}$ luminosities, there is no significant difference in the distributions of the CPG and KI integrated luminosities. Haynes and Herter (1988) found a similar null result when examining the 60 and $100 \mu \mathrm{m}$ emission from widely separated CPG pairs.

The presence of a nearby companion does have some influence on the integrated infrared properties of a galaxy, however, as shown in Figure 4. The distribution of the ratio of infrared-to-optical luminosities of CPG systems ranges extends nearly an order of magnitude higher than for $\mathrm{KI}$ galaxies. If the ratio $\mathrm{L}_{\mathrm{ir}} / \mathrm{L}_{\mathrm{opt}}$ can be taken in part as a measure of the star formation rate in a galaxy, then on average, close pairs exhibit only a slight enhancement in galaxy-wide star forming activity. This concurs with the findings of Kennicutt et al. (1987) who found only a mild enhancement in disk star formation in their sample of interacting pairs. Interestingly, the CPG galaxies with the highest values of $\mathrm{L}_{\mathrm{ir}} / \mathrm{L}_{\mathrm{opt}}$ are not the most luminous cases.

CPG and $\mathrm{KI}$ galaxies span a similar range in mid- and far infrared colors, as is shown in Figure 5. The CPG source do exhibit a slightly larger scatter in each color, reminiscent of the larger scatter in optical colors found in interacting galaxies by Larson and Tinsley (1978). We do not find evidence for systematically higher color temperatures among close pairs, as was reported by Telesco, Wolstencroft and Done (1988) for pairs drawn from the Arp Atlas. In addition, we have been unable to find any correlation between the infrared luminosity, $\mathrm{L}_{\mathrm{ir}} / \mathrm{L}_{\mathrm{opt}}$, or infrared color of the CPG pairs and pair separation, relative size or relative velocities. If outright collisions or mergers lead to extremely luminous infrared emission (e.g. Sanders et al. 1988), our analysis may be expected to miss such sources since the CPG is biased against very close pairs and mergers.

There does appear to be a weak dependence of $\mathrm{L}_{\mathrm{ir}}$ on interaction morphology, as is shown in Figure 6, which is also present in $\mathrm{L}_{\mathrm{ir}} / \mathrm{L}_{\text {opt }}$ (not shown). If the interaction morphology can be interpreted as an approximate age indicator of the encounter through comparison with numerical simulations, the SEP systems should be the youngest (no sign of disturbance) or possibly the oldest, followed by the disturbed pairs (DIS), the LIN pairs those showing linear bridges or tails, and finally the pairs enshrouded by common envelopes (ATM). The median luminosities and colors then show when the induced star formation in each class must reach its peak. Considerable dispersion is introduced due to the different morphological types involved in the interactions, uncertain viewing geometries, and coarse morphological classifications. One means of clarifying this relationship may be to correlate the ages of interactions deduced from numerical simulations to ages from starburst models. 


\section{REFERENCES}

Cutri, R.M. and McAlary, C.W. 1985, Ap.J., 296, 90.

Haynes, M.P. and Herter, T. 1988, A.J., 96, 504.

Karachentsev, I.D. 1972, Comm.Spec.Astrofiz.Obs., 7, 1.

Karachentseva, V.E. 1973, Comm.Spec.Astrofiz.Obs., 8, 1.

Keel, W.C., Kennicutt, R.C., Hummel, E. and van der Hulst, J.M. 1985, A.J., 90,708.

Kennicutt, R.C., Keel, W.C., van der Hulst, J.M., Hummel, E. and Roettiger, K.A. 1987, A.J., 93, 1011.

Larson, R.B. and Tinsley, B.M. 1978, Ap.J., $219,46$.

Rieke, G.H. 1976, Ap.J.(Letters), 206; L15.

Sanders, D.B., Soifer, B.T., Elias, J.H., Madore, B.F., Neugebauer, G. and Scoville, N.Z. 1988, Ap.J., $325,74$.

Telesco, C.M., Wolstencroft, R.D. and Done, C. 1988, Ap.J., 329, 174.
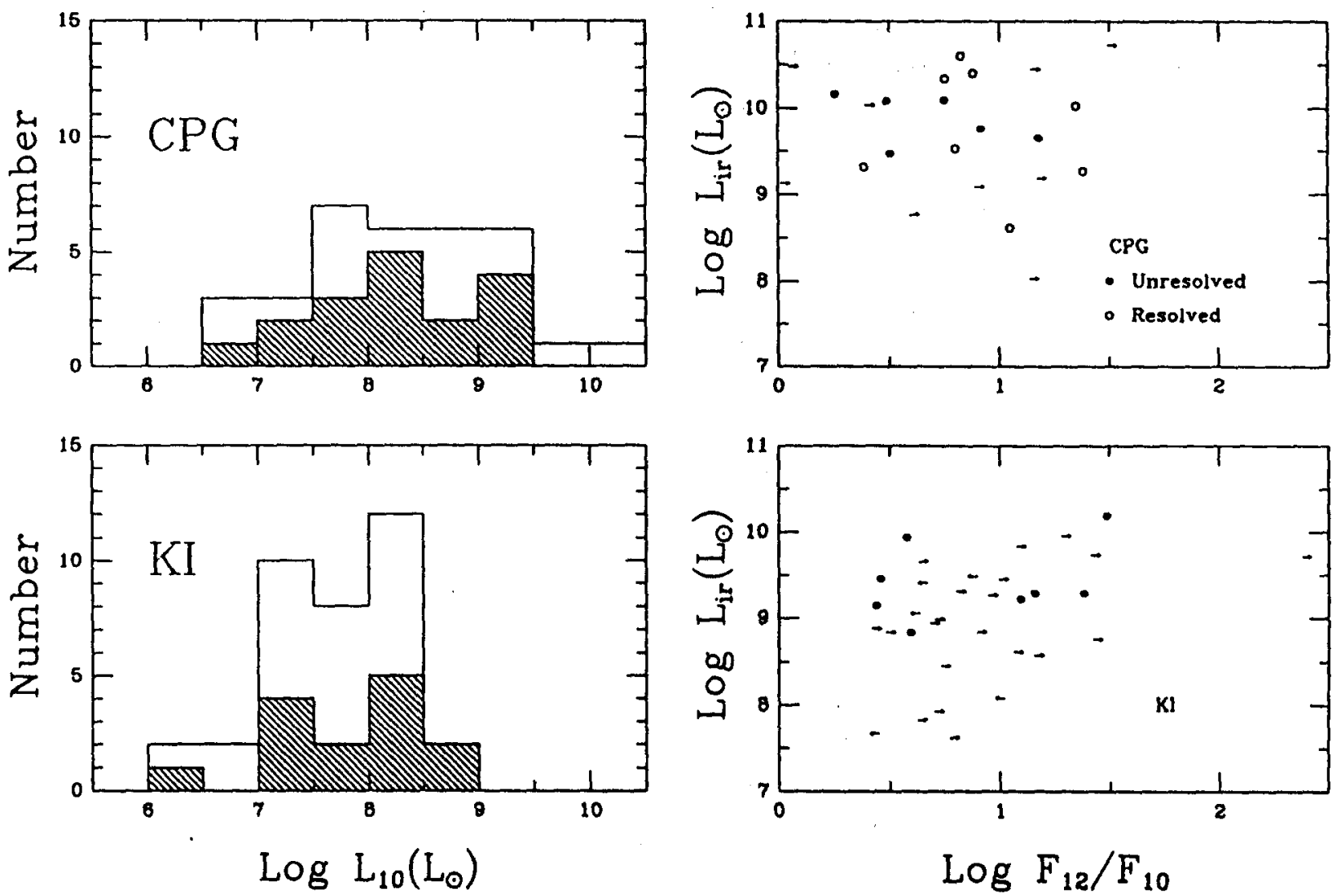

Figure 1 - The $10 \mu \mathrm{m}$ luminosity distribution of CPG galaxies (top) and KI galaxies (bottom). Open boxes represent $3 \sigma$ upper limits to luminosities.

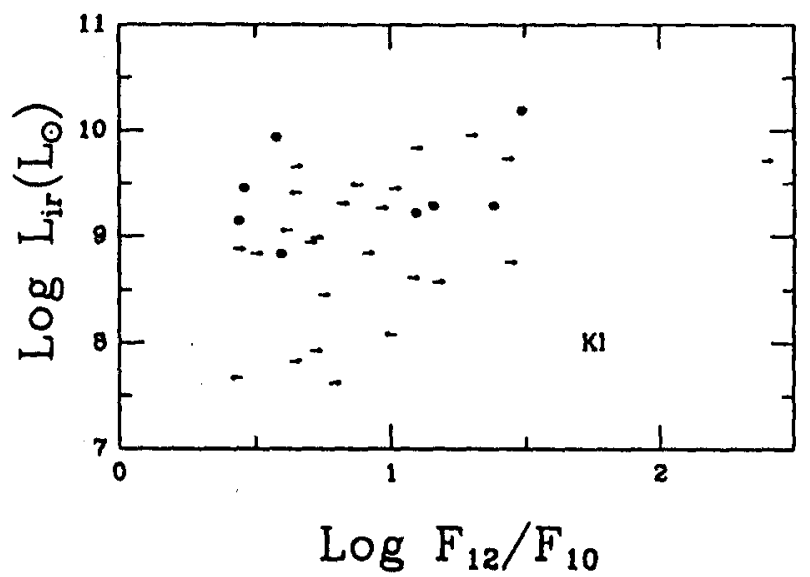

Figure 2 - The ratio of large beam IRAS $12 \mu \mathrm{m}$ flux densities to small aperture ground based $10.4 \mu \mathrm{m}$ flux densities. Upper and lower limits are denoted by arrows. 

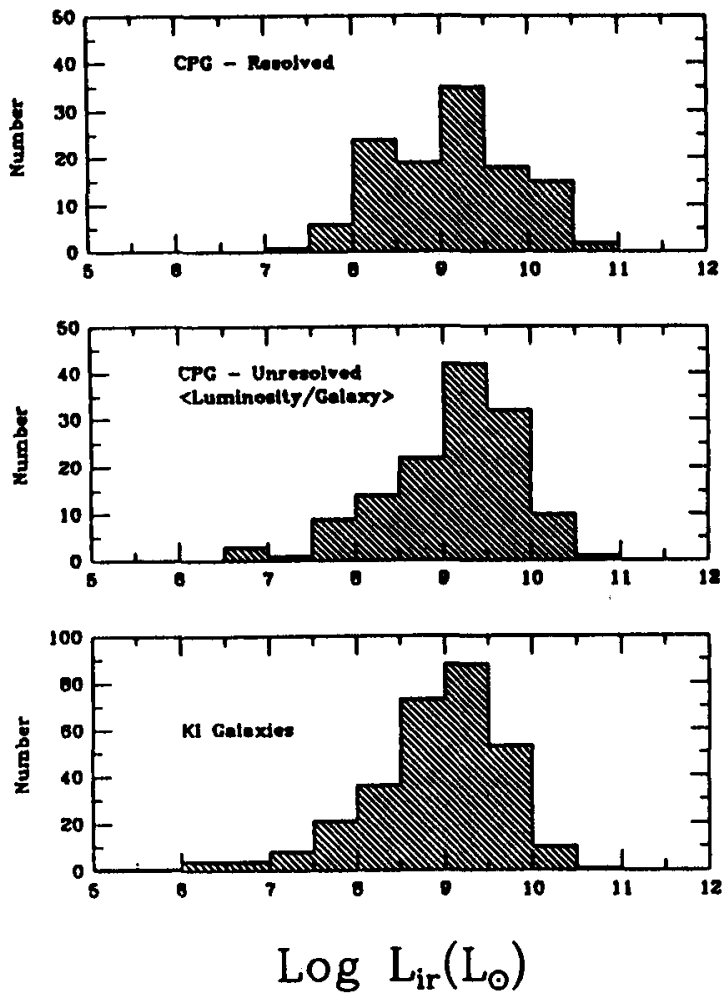

Figure 3 - The integrated 12-100 $\mu \mathrm{m}$ luminosities for CPG and KI galaxies calculated using IRAS ADDSCAN data. For unresolved CPG pairs (center) the average luminosity per galaxy is displayed.
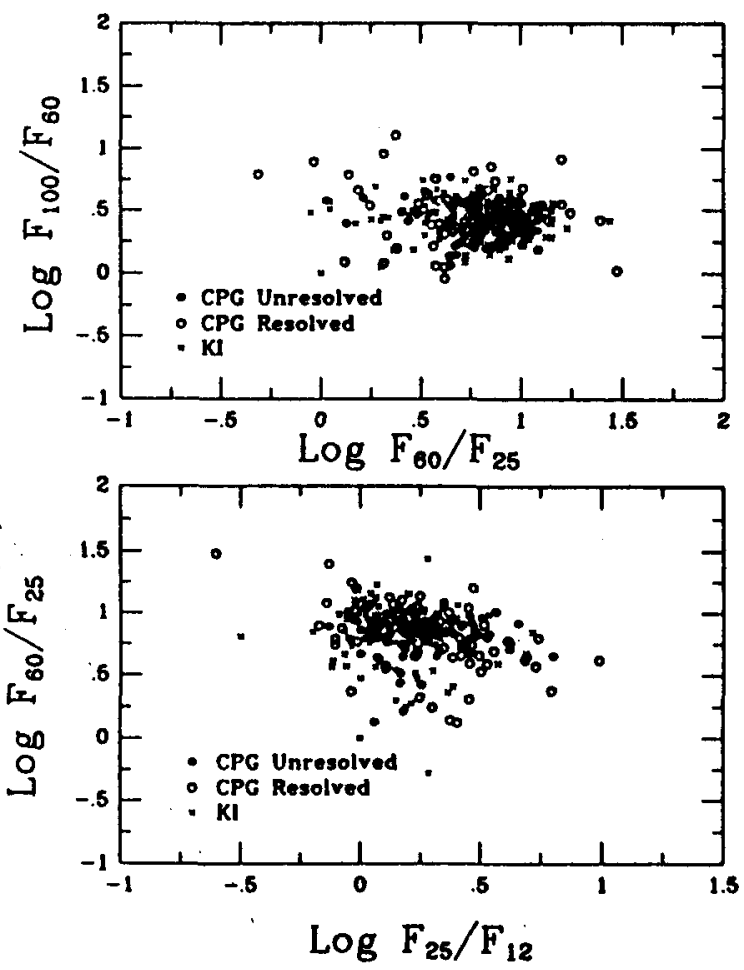

Figure 5 - The mid-and far-infrared colors of CPG and $\mathrm{KI}$ galaxies taken from IRAS ADDSCAN data.

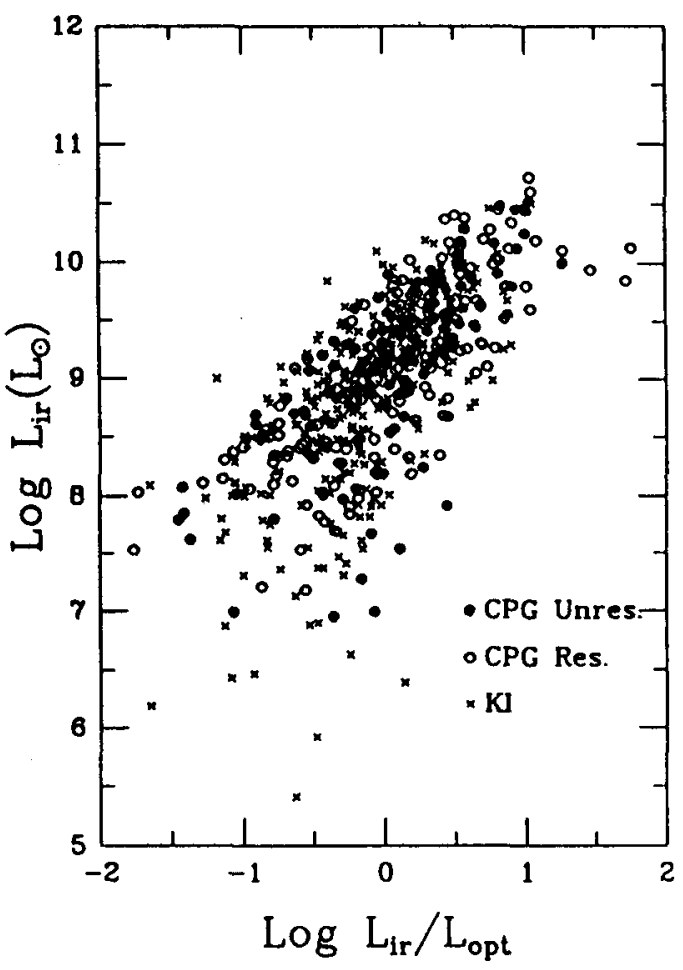

Figure 4- The integrated infrared luminosity of CPG and $\mathrm{KI}$ galaxies as a function of infrared-to-optical luminosity ratio.
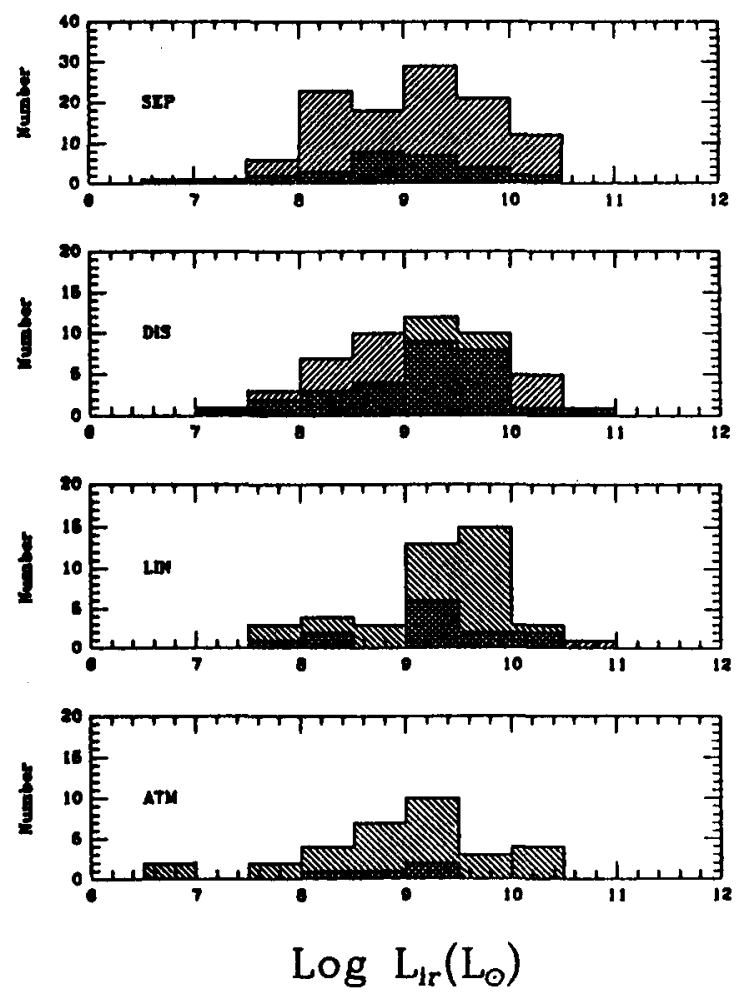

Figure 6 - The integrated infrared luminosity distribution for each class of CPG interaction morphology. Luminosities for resolved pairs are shaded with IIII, and unresolved pairs with IIII 